\title{
Imagem no traumatismo craniano
}

\author{
Imaging of traumatic brain injury
}

\section{Gabriel Scarabôtolo Gattás}

Gattás GS. Imagem no traumatismo craniano / Imaging of traumatic brain injury. Rev Med (São Paulo). 2011 out.dez.;90(4):157-68.

RESUMO: A avaliação por imagem do traumatismo cranioencefálico (TCE) tem tido importante papel na determinação do pronto diagnóstico de lesões traumáticas intracranianas, definindo conduta terapêutica clínica ou cirúrgica emergencial, posteriormente auxiliando na monitorização terapêutica e permitindo acesso a estimativa mais precisa do prognóstico do paciente. A tomografia computadorizada (TC) é o método por imagem mais adequado no atendimento inicial ao paciente vítima de TCE detectando rapidamente lesões potencialmente fatais que necessitem de tratamento cirúrgico imediato. A angiotomografia permite diagnosticar lesões vasculares crânio-cervicais potencialmente graves. Após a estabilização clínica destes pacientes a ressonância magnética (RM) contribui no aumento da detecção e na melhor caracterização das lesões traumáticas encefálicas e de suas possíveis complicações tais como herniações cerebrais e lesões isquêmicas. Neste artigo serão abordadas as principais lesões traumáticas primárias e secundárias cranioencefálicas e suas principais características à TC e RM, métodos que fornecem importantes informações diagnósticas e prognósticas nos pacientes vítimas de TCE.

DESCRITORES: Diagnóstico por imagem; Traumatismos craniocerebrais; Traumatismos do sistema nervoso; Imagem por ressonância magnética; Tomografia computadorizada por raios $\mathrm{X}$.
ABSTRACT: The imaging evaluation of traumatic brain injury (TBI) has played an important role in determining the early diagnosis of traumatic intracranial injury, defining therapeutic medical or surgical emergency, subsequently assisting in monitoring therapy and allowing access to more accurate estimate of the patient's prognosis. Computed tomography (CT) imaging is the method most appropriate to the patient in the initial care of TBI victim quickly detecting life-threatening injuries requiring immediate surgical treatment. The angiotomography (CTA) allows diagnosing craniocervical vascular lesions potentially serious. After clinical stabilization of these patients magnetic resonance imaging (MRI) contributes to increased detection and better characterization of traumatic brain injuries and its possible complications such as cerebral herniation and ischemic lesions. In this article we will discuss the major primary and secondary traumatic cranioencephalic injuries and its main features on CT and MRI methods that provide important diagnostic and prognostic information in patients victims of TBI.

KEYWORDS: Imagind diagnostic; Craniocerebral trauma; Trauma, nervous system; Magnetic resonance imaging; Tomography, X-ray computed.

Médico, Instituto de Radiologia do Hospital das Clínicas da Faculdade de Medicina da Universidade de São Paulo. Endereço para correspondência: InRad-HCFMUSP. Av. Dr. Eneas de Carvalho Aguiar, 255. São Paulo, SP, CEP: 05403-001. e-mail: gsgattas@gmail.com 


\section{INTRODUÇÃO}

W a avaliação inicial de um paciente vítima existem questões fundamentais que devem ser rapidamente respondidas: se há lesão traumática intracraniana, qual sua natureza e qual seu tratamento, se clínico ou cirúrgico emergencial.

Neste sentido, a avaliação por imagem do TCE tem tido importante papel na determinação do pronto diagnóstico de lesões traumáticas intracranianas, definindo conduta terapêutica clínica ou cirúrgica emergencial, posteriormente auxiliando na monitorização terapêutica e permitindo acesso a estimativa mais precisa do prognóstico do paciente.

A radiografia simples de crânio, a tomografia computadorizada (TC), a ressonância magnética (RM) e a angiografia digital são os principais métodos por imagem utilizados nestes pacientes. A radiografia simples de crânio não é capaz de excluir lesão intracraniana, tendo elevados índices falso-negativos. Inversamente, a presença de fratura craniana à radiografia simples não necessariamente indica presença de lesão intracraniana ${ }^{1,2}$. Portanto, não é um método que deve ser utilizado para o rastreamento de lesão traumática intracraniana.

A TC é o método por imagem mais adequado no atendimento inicial ao paciente vítima de TCE, pois, quando comparada à RM, é mais disponível, tem menor custo, e tem ótima sensibilidade na detecção das principais lesões traumáticas intracranianas agudas, permitindo rapidamente a identificação de lesões potencialmente fatais que necessitem de tratamento cirúrgico imediato ${ }^{3}$. Os tomógrafos de multidetectores (TC "multislice") são extremamente rápidos, ideais para pacientes agitados, com confusão mental, em intoxicação exógena e em crianças, situações freqüentes nos pacientes com TCE. Além disso, a TC "multislice" permite avaliação multiplanar e tridimensional das estruturas ósseas cranianas, da base do crânio e da coluna cervical, bem como estudo das estruturas vasculares por angiotomografia (angio-TC) trazendo grande avanço no diagnóstico das lesões dos vasos cervicais e intracranianos, seja nos traumas fechados ou nos perfurantes. Assim, em poucos segundos pode-se ter o diagnóstico não só das clássicas lesões traumáticas intracranianas, mas também das lesões traumáticas da base do crânio, incluindo-se as estruturas das mastóides, da face e coluna cervical bem como dos vasos cervicais e intracranianos.

A RM mostra excelente definição das estruturas encefálicas, muito superiores em relação à TC, gerando imagens multiplanares, sendo um método mais sensível que a TC para a detecção de lesões intracranianas, especialmente aquelas não hemorrágicas, hemorrágicas de pequeno volume, ou de localização profunda ou infratentoria| ${ }^{3-7}$. Porém a RM tem algumas restrições que impossibilitam sua larga utilização no atendimento inicial à vítima de TCE, dentre elas, seu alto custo, menor disponibilidade, maior tempo de execução do exame e elevado campo magnético dificultando o manejo destes pacientes que freqüentemente trazem consigo corpo estranho metálico perfurante ou dispositivos metálicos de monitorização e suporte vital.

Após a estabilização clínica destes pacientes e já se tendo informações adicionais obtidas pela TC (e eventualmente angio-TC) do atendimento inicial, a RM contribui no aumento da detecção e na melhor caracterização das lesões traumáticas encefálicas e de suas possíveis complicações tais como herniações cerebrais e lesões isquêmicas. O protocolo de RM preconizado para a adequada avaliação do TCE inclui as sequências T1 e T2 spin-eco, T2* (gradiente eco), "fluid attenuated inversion recovery" (FLAIR), Difusão e mais recentemente, sequência de suscetibilidade magnética (SWI), que quando analisadas conjuntamente, permitem caracterizar maior número de lesões traumáticas encefálicas, mesmo que de pequenas dimensões, não hemorrágicas ou em fossa posterior, além de infartos agudos, herniações cerebrais, hemorragia subaracnóidea, e hematomas extra e subdurais mesmo que pequenos e de fina espessura, inclusive aqueles em fossa posterior. Tendo mais sensibilidade diagnóstica, a RM assume importante papel nesta segunda avaliação por imagem do TCE, trazendo mais detalhamento das lesões e maior precisão no estadiamento, contribuindo na conduta terapêutica e no prognóstico destes pacientes ${ }^{8-10}$.

\section{LESÕES TRAUMÁTICAS PRIMÁRIAS}

\section{Lesões superficiais extracranianas e fraturas cranianas}

As fraturas cranianas estão presentes em 50\% a $60 \%$ dos pacientes com TCE grave, dividindo-se em três tipos principais: linear, complexas ou com afundamento, e de base de crânio.

A fratura craniana mais freqüente é linear e sem desalinhamentos, freqüentemente associada à presença de hematoma extradural (Figura 1). 

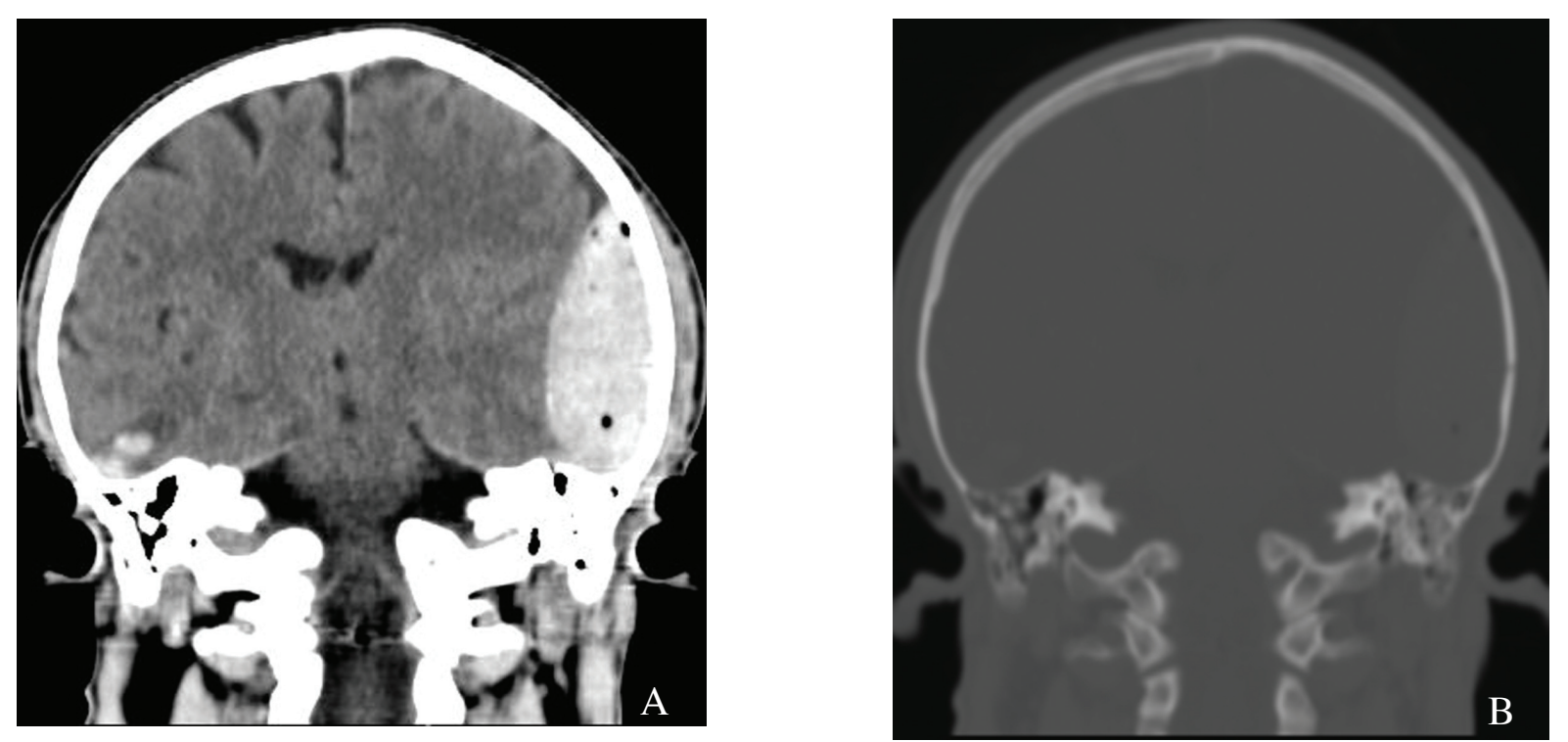

Figura 1. Fratura craniana sem desalinhamento - reconstrução coronal. (A) Janela para avaliação do parênquima encefálico e (B) janela óssea demonstrando hematoma extradural adjacente à fratura. Note contusão cortical hemorrágica temporal contralateral (lesão golpe-contragolpe)

As fraturas com afundamento podem ocasionar agressão ao córtex cerebral, podendo ser necessária exploração cirúrgica, principalmente se o afundamento for maior que $5,0 \mathrm{~mm}$ ou se a depressão for maior que a espessura da calota craniana (Figura 2) ${ }^{11-13}$. A TC "multislice" com reconstruções multiplanares e tridimensionais permite excelente avaliação das fraturas crânio-faciais graves, evidenciando com muita precisão seus múltiplos traços, desalinhamentos e afundamentos, sendo importante no planejamento da exploração cirúrgica (Figura 3 ).

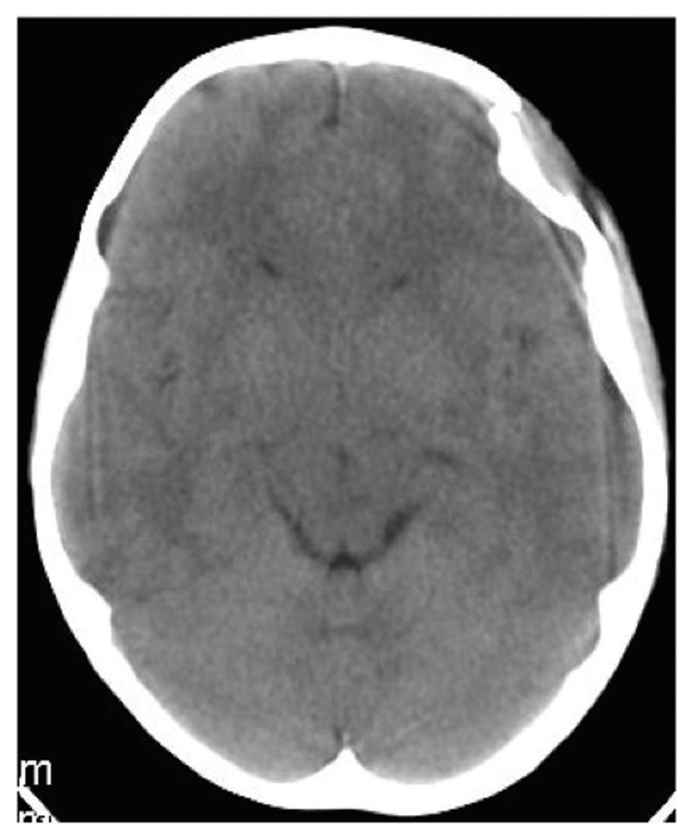

Figura 2. TC evidenciando fratura-afundamento da calota craniana frontal esquerda
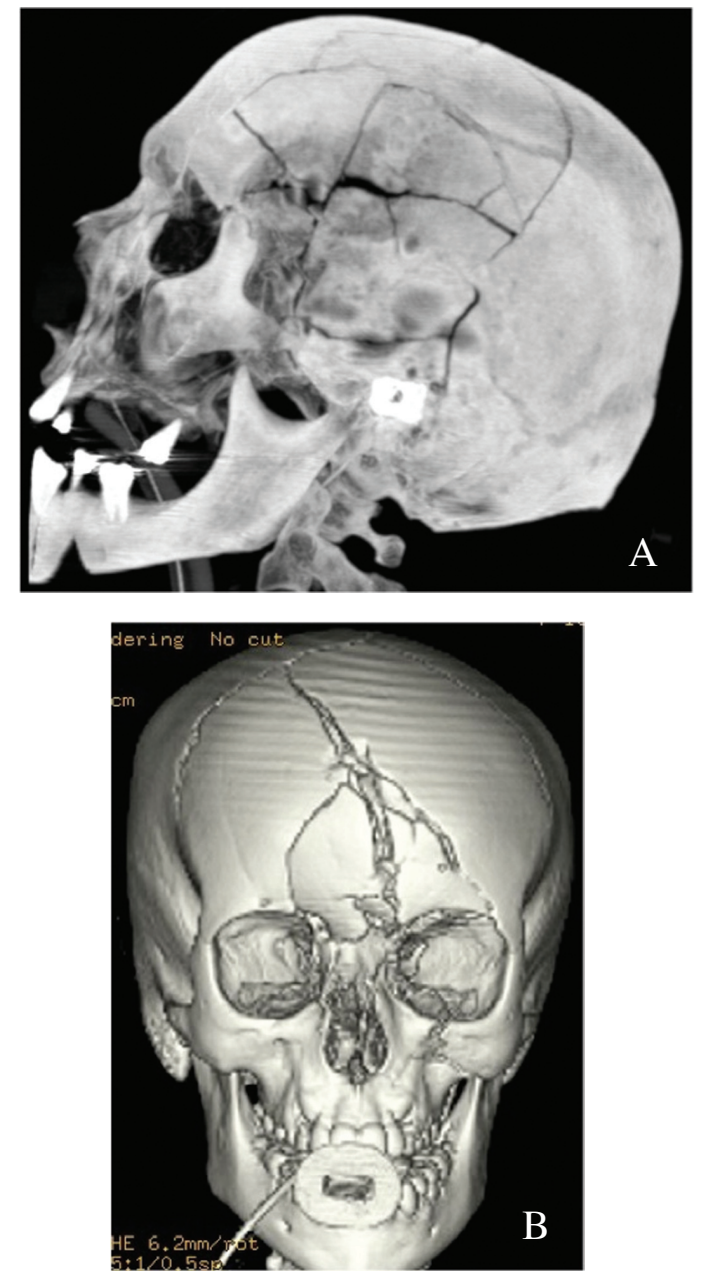

Figura 3. Múltiplas fraturas crânio-faciais complexas e desalinhadas. TC "multislice" com reconstrução "maximal intensity projection" (MIP) sagital (A) e tridimensional (B) 
Da mesma forma, a TC "multislice" permite excelente avaliação de importantes estruturas ósseas das mastóides e da base do crânio tais como os canais carotídeos, forames transversos das artérias vertebrais, forames jugulares, podendo sinalizar a possibilidade de lesões vasculares (Figura 4).
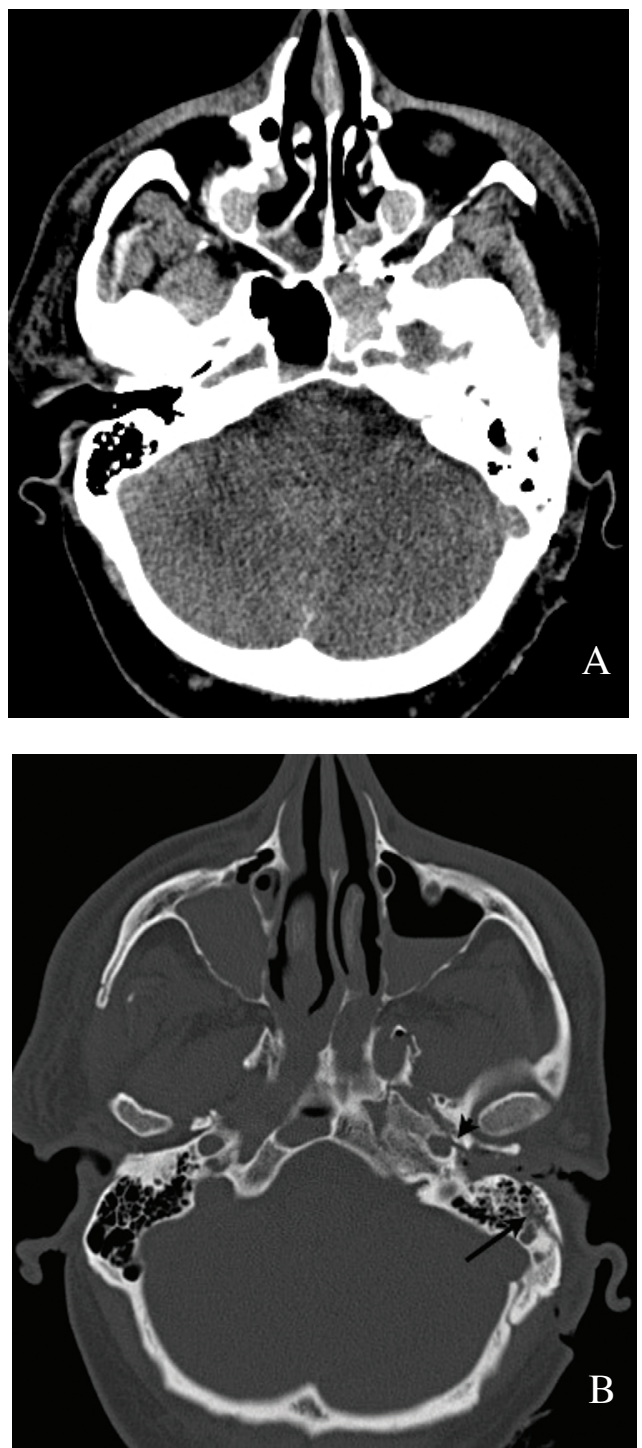

Figura 4. TC: (A) hemossinus esfenoidal e maxilar bilateral. (B) Fratura longitudinal na mastóide esquerda (seta), com velamento de suas células; pequeno traço de fratura na parede lateral do canal carotídeo esquerdo (cabeça de seta)

Nos planos superficiais pode-se observar lacerações, ferimentos corto-contusos, orifícios de entrada e saída de ferimentos por arma de fogo e hematomas subgaleais (Figura 5), geralmente de pouco significado clínico maior, à exceção dos processos infecciosos que aí podem se desenvolver e dos ferimentos perfurantes que podem causar pseudoaneurismas ou fístulas arteriovenosas ${ }^{14}$.

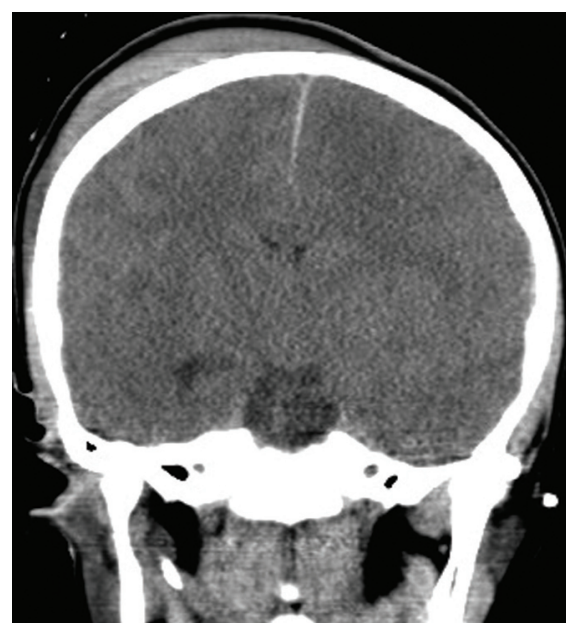

Figura 5. Reconstrução coronal de TC "multislice": hematoma subgaleal

\section{Ferimentos por arma de fogo e outros ferimentos penetrantes}

Ferimento por arma de fogo (FAF) de trajeto unilobar, em geral, tem melhor prognóstico que aqueles de trajeto bi-hemisférico, transventricular ou cursando pela fossa posterior. A TC tem importante papel na avaliação inicial destes pacientes, caracterizando a localização e o trajeto do projétil, demonstrando a localização e a extensão do ferimento encefálico, o grau de hemorragia intracraniana e de fragmentação do projétil, presença de fraturas associadas, e, principalmente, mostrando se há lesões cirurgicamente tratáveis tais como hematomas intracranianos ou herniação cerebral. A alta densidade metálica do projétil ou de seus fragmentos gera intensos artefatos que prejudicam a avaliação do parênquima encefálico adjacente, neste aspecto sendo uma limitação da TC (Figura 6).

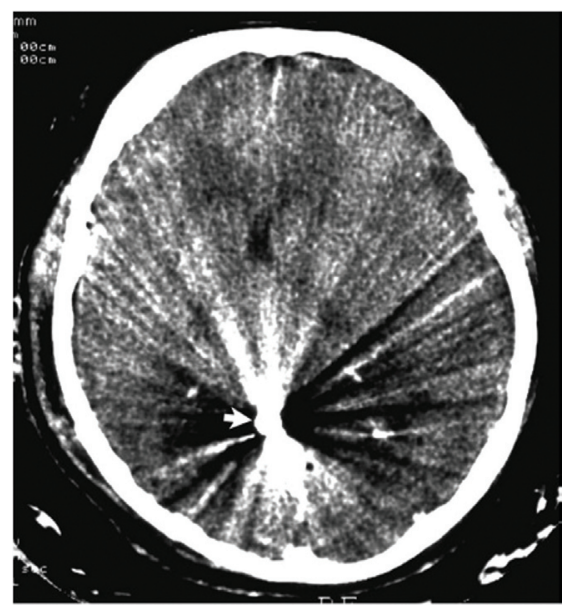

Figura 6. TC: Ferimento por arma de fogo (FAF) na admissão do pronto-atendimento. TC: Note projétil de arma de fogo gerando intensos artefatos que prejudicam a avaliação do parênquima encefálico 
A TC também é bastante útil na avaliação de outros ferimentos penetrantes tais como por arma branca ou outros objetos perfurantes (Figura 7). Qualquer ferimento penetrante tem potencial para causar lesão vascular tais como laceração, dissecção, pseudoaneurisma, fístula arteriovenosa e trombose venosa, neste caso sendo válido considerar a necessidade de avaliação por angiografia digital (Figura 7) que é método padrão-ouro, ou mais recentemente como alternativa não invasiva, por angio-TC que além do estudo vascular, permite avaliação multiplanar e 3D do crânio, contribuindo no planejamento cirúrgico dos ferimentos penetrantes. A angio-RM em geral é contra-indicada devido à presença de corpo estranho metálico ${ }^{15-17}$.
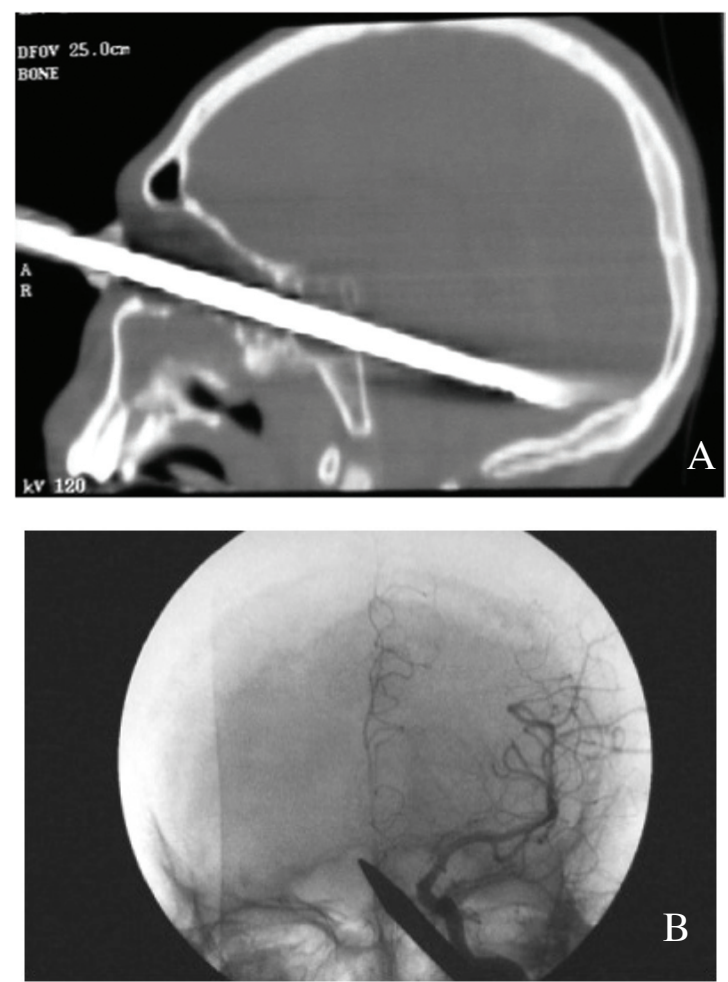

Figura 7. TC: (A) Reconstrução sagital de ferimento penetrante por chave de fenda de curso intraorbitário, paraclinóide e em fossa posterior, em estreita proximidade com o canal carotídeo. (B) arteriografia digital excluindo lesão vascular

\section{Hemorragias extra-axiais traumáticas}

Os principais tipos de hemorragia extra-axial traumática são: hematoma extradural, hematoma subdural, hemorragia subaracnóidea e hemorragia intraventricular, todas elas detectáveis com alta sensibilidade pela TC devido à elevada atenuação do sangue na fase aguda, sendo geralmente hiperatenuantes em relação às demais estruturas encefálicas. Por isso, a TC de crânio sempre deve ser analisada em três janelas fundamentais: janela para avaliação do parênquima encefálico, janela para detecção de hemorragias hiperdensas e janela para avaliação óssea (Figura 8) pois sua análise somente pela janela para parênquima encefálico pode ser insuficiente para detectar fraturas cranianas e pequenos hematomas extra-axiais hiperdensos que se confundem com a hiperatenuação da calota craniana. A RM é superior à TC nas hemorragias de pequeno volume (por exemplo, hematomas laminares), naquelas numa fase evolutiva subaguda ou em pacientes anêmicos, quando a atenuação do sangue se assemelha ao do parênquima cerebral à $\mathrm{TC}$, e naquelas localizadas na fossa posterior ${ }^{3}$.

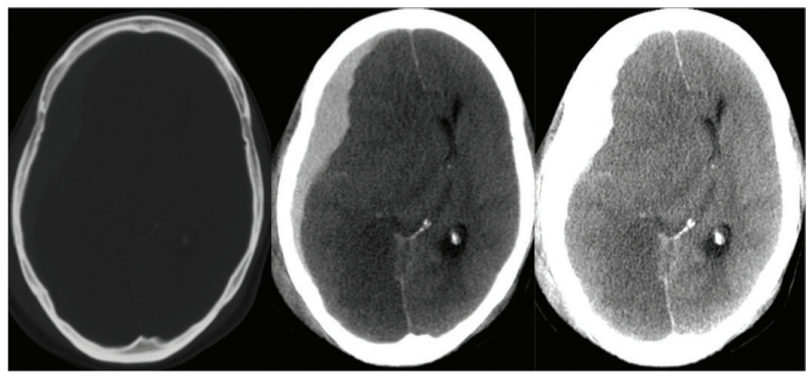

Figura 8. TC: TCE grave com hematoma subdural agudo à direita. Janela para avaliação óssea, assegurando pesquisa eficaz de fraturas cranianas (imagem da esquerda). Janela mais aberta para detecção de hemorragias agudas (imagem ao centro), note hematoma subdural hiperdenso direito determinando herniação uncal e subfalcina para a esquerda. Janela para avaliação de parênquima encefálico (imagem da direita), note hipoatenuação cortico-subcortical occipital direita compatível com infarto cerebral traumático secundário

Hematoma extradural (HED): é uma coleção hemorrágica localizada entre a tábua interna da calota craniana e a duramáter, no espaço extradural. Está presente entre $2 \%$ e $12 \%$ dos TCEs, num pico de faixa etária entre 10 e 30 anos, sendo menos comum em crianças e idosos. A apresentação clássica caracterizada pelo intervalo lúcido com perda tardia de consciência somente é observada numa minoria destes pacientes, não havendo perda de consciência em mais de $50 \%$ dos casos. A maioria dos hematomas extradurais tem sua origem a partir de uma fratura craniana lacerando ramos arteriais meníngeos ou seios venosos durais, geralmente unilaterais, supratentoriais, e nas regiões têmporoparietais (Figuras 1 e 9).

Os hematomas extradurais de origem venosa são menos freqüentes que os arteriais, em geral decorrentes de fraturas que lesam a parede de um seio venoso dural. Sua localização mais comum é na fossa posterior, por lesão do seio transverso ou do seio sigmóide, e na fossa cerebral média por lesão do seio esfenoparietal|18,19, e mais raramente 
no vértice craniano por lesão do seio sagital superior (Figura10) $^{20,21}$.

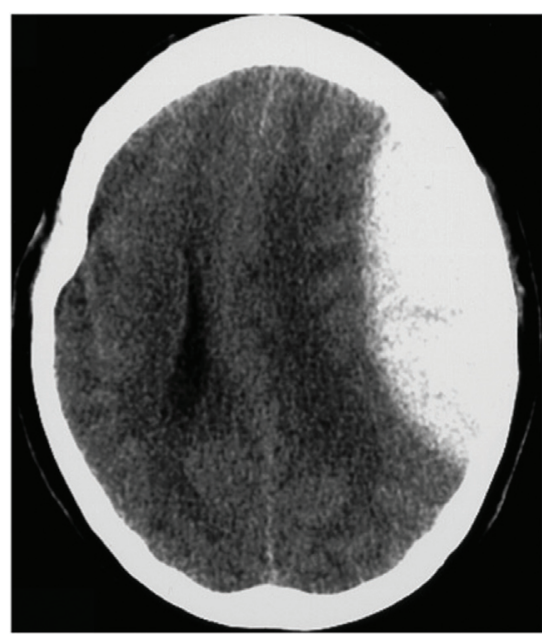

Figura 9. TC: Hematoma extradural agudo hiperdenso à esquerda, de morfologia biconvexa, determinando compressão ventricular e desvio da linha mediana para a direita

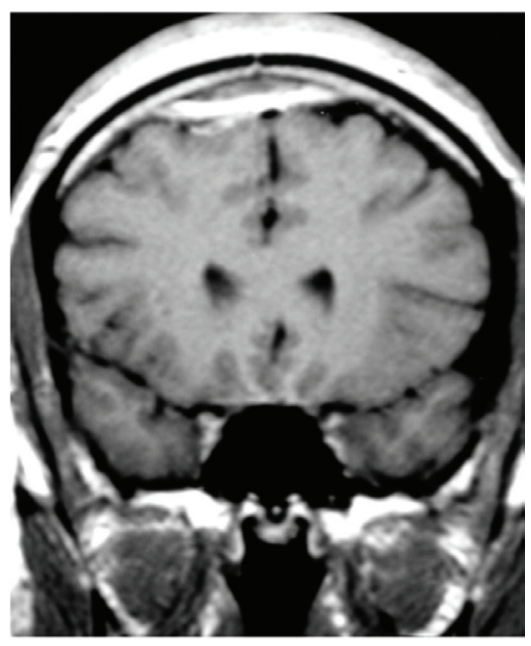

Figura 10. RM: Seqüência coronal pesada em T1 evidencia hematoma extradural de vértice craniano afastando o seio sagital superior da tábua interna da calota craniana

Classicamente o hematoma extradural se apresenta à TC como uma coleção extra-axial que afasta o córtex da tábua interna da calota craniana, tendo formato de uma lente biconvexa homogeneamente hiperatenuante que, quando de maiores dimensões, afasta e abaula a convexidade cortical causando compressões ventriculares, herniações cerebrais e freqüentemente associa-se a contusões corticais adjacente ou de contragolpe ${ }^{17}$ (Figuras 1 e 9). Devido à grande aderência da duramáter na tábua interna craniana no curso das suturas, os HEDs em geral não ultrapassam as linhas de sutura, diferentemente dos hematomas subdurais que atravessam livremente as linhas de sutura (Figura 8).
Os pequenos hematomas extradurais que cursam paralelos ao plano de corte axial da TC de crânio convencional podem não ser detectados tais com aqueles do assoalho das fossas cerebrais anteriores e médias e do vértice craniano, e nestes casos a RM e a TC "multislice" com reconstruções multiplanares são métodos mais sensíveis para sua detecção (Figura 10) 20, 21.

Hematoma subdural: localiza-se no espaço subdural, mais freqüentemente no compartimento supratentorial (95\%), em geral ao longo das convexidades corticais, da foice cerebral ou da tenda cerebelar, freqüentemente causando acentuados desvios das estruturas encefálicas da linha mediana e herniações cerebrais ${ }^{12,17}$. Hematomas bilaterais, ao longo da foice cerebral ou da tenda cerebelar são comuns na síndrome da criança espancada ${ }^{12,19,22,23}$. Está presente em mais de $30 \%$ dos TCEs graves, sendo que em idosos está frequentemente associado à quedas e traumas leves, e em adultos jovens está associado à acidentes automobilísticos e traumas mais violentos ${ }^{12,24}$.

O hematoma subdural ocorre na vigência de forças de aceleração/desaceleração que causam laceração de veias corticais enquanto cursam pelo espaço subdural, antes de drenarem ao seio venoso dural adjacente. Estas veias são particularmente suscetíveis a este mecanismo de trauma uma vez que de um lado elas estão aderidas ao seio venoso dural ancorado na calota craniana e do outro, ao encéfalo que se movimenta durante a ação destas forças traumáticas, ocasionando estiramento, laceração ou ruptura das mesmas ${ }^{19,25}$.

O hematoma subdural agudo classicamente apresenta-se à TC como uma coleção hiperatenuante de morfologia semelhante a uma lente côncavo-convexa, com sua face côncava moldandose à convexidade cortical, foice cerebral ou tenda cerebelar, podendo atravessar as linhas de suturas mas não as inserções durais ou a foice cerebral (Figura 8) 3-5,17,19,23,26.

Os hematomas subdurais subagudos ou em pacientes com anemia severa podem ter a mesma atenuação que o parênquima encefálico (isoatenuantes), nestes casos sendo de difícil diagnóstico. Assim, sinais que sugiram sua presença tais como apagamento regional dos sulcos corticais, sulcos corticais que não atingem a tábua interna do crânio, desvio da interface substância branca/ cinzenta cortical, compressão do ventrículo lateral e desvio da linha mediana devem ser valorizados e indicar prosseguimento na investigação por imagem, seja por TC com contraste endovenoso permitindo uma nítida diferenciação entre o parênquima encefálico contrastado e o hematoma subdural 
isodenso que não apresenta realce pós-contraste, ou pela RM, método bastante sensível na detecção destes hematomas independentemente de sua fase evolutiva (Figura 11) 4,11,17,27-29.

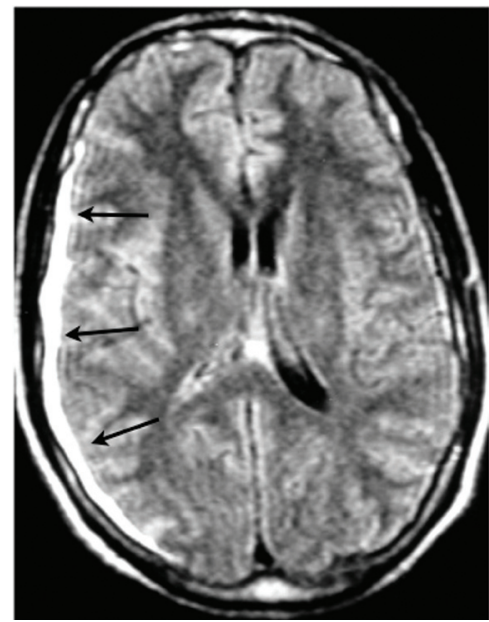

Figura 11. RM: Delgado hematoma subdural à direita, exibindo hipersinal na sequência FLAIR (setas)

Os hematomas subdurais crônicos estáveis classicamente se apresentam como uma coleção hipoatenuante homogênea à TC. Entretando, quando ocorrem novos sangramentos no interior de um HSD crônico, forma-se nível líquido-líquido constituído por componente hipoatenuante hemorrágico antigo e pelo novo componente hemorrágico hiperdenso que tende a assumir uma posição decúbito-dependente (Figura 12). Além disso, HSDs crônicos podem desenvolver membranas em seu interior conferindo aspecto multiloculado ou trabeculado com compartimentos tendo diferentes características de atenuação ${ }^{11}$.

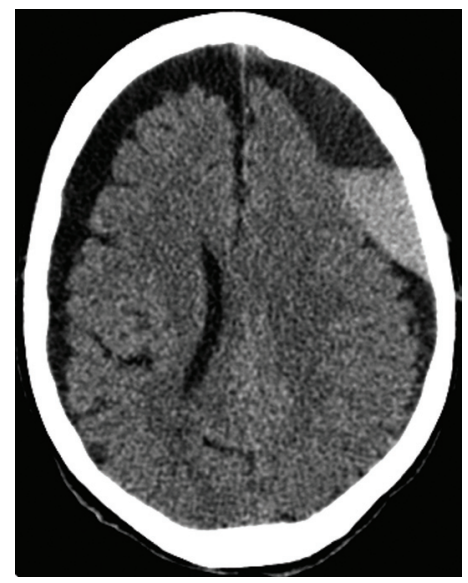

Figura 12. TC: Hematoma subdural crônico hipoatenuante bilateral, sendo que à esquerda observa-se sangramento recente hiperatenuante formando nível líquido-líquido

Hemorragia subaracnóidea traumática: caracteriza-se pelo extravasamento de sangue no espaço subaracnóide devido a laceração de artérias ou veias corticais que cursam por este espaço, ou por extensão de hemorragia proveniente de contusões corticais. Está presente na maioria dos casos de TCEs moderados ou graves e classicamente se localiza na cisterna interpeduncular ou silviana ${ }^{30}$ mas podendo ocorrer em qualquer região do espaço subaracnóide. Na TC é identificada como conteúdo hiperatenuante preenchendo as cisternas e os sulcos corticais (Figura 13 e 21). A quantidade da hemorragia subaracnóidea deve ser correlacionada com a severidade do TCE, e, havendo alguma desproporção, deve-se suspeitar de lesão vascular maior associada, neste caso sendo válido o prosseguimento na investigação diagnóstica através de angio-TC ou arteriografia digital ${ }^{11,12,17}$. RM, principalmente através das seqüências $\mathrm{T}^{*}$ gradiente eco e FLAIR também é um método bastante sensível à detecção de hemorragia subaracóidea traumática, porém a TC é mais sensível ${ }^{11}$.

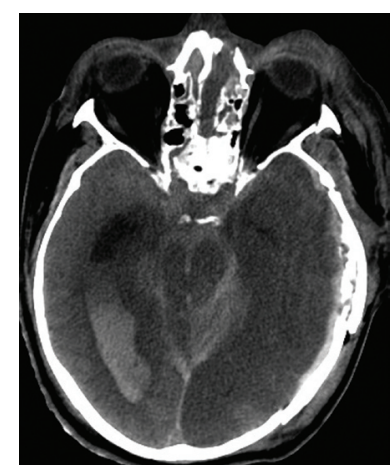

Figura 13. TC: Hemorragia subaracnóidea traumática aguda caracterizada por conteúdo hiperatenuante ocupando os sulcos corticais e as cisternas da base, mais notadamente nas cisternas interpeduncular e perimesencefálica. Hemoventrículo volumoso observado na ventrículo lateral direito

Hemorragia intraventricular traumática: pode ser causada por laceração de veias ependimárias ou resultar da extensão de um hematoma intraparenquimatoso ou de uma hemorragia subaracnóidea através do IV ventrículo. Comumente associa-se a lesão axonal difusa (LAD) e a hemorragia intraparenquimatosa cerebral, da substância cinzenta profunda ou do tronco cerebral, sendo rara como achado isolado na ausência de outras hemorragias intracranianas (Figuras 13 e 18) ${ }^{17}$.

\section{Lesões traumáticas encefálicas}

Lesão axonal difusa (LAD): é uma das lesões mais freqüentemente encontradas em pacientes vítimas de TCEs graves, constituindo cerca de $50 \%$ das lesões primárias encefálicas, tendo uma taxa de mortalidade associada estimada em 10\%5,12,19. Classicamente, o paciente acometido por este tipo de lesão apresenta acentuado rebaixamento do nível de consciência ${ }^{12}$. O mecanismo de trauma baseia- 
se na diferença de movimentação entre estruturas encefálicas de diferentes densidades durante a ação de uma abrupta força de aceleração/desaceleração fazendo com que haja estiramento, cisalhamento e rotura de fibras axonais e de pequenos vasos sanguíneos perfurantes existentes na interface entre estas estruturas. Assim, além da lesão axonal, há lesão vascular responsável pela presença de hemorragia associada. As lesões da LAD geralmente são múltiplas, ovais ou elípticas, tendo o maior eixo paralelo ao plano do trato axonal lesionado, medem entre 5 e $15 \mathrm{~mm}$, sendo geralmente menores nas regiões mais periféricas, e maiores nas regiões mais profundas. As localizações mais freqüentes da LAD são: substância branca subcortical dos hemisférios cerebrais (principalmente na interface entre a substância branca e cinzenta), corpo caloso, região dorsolateral do mesencéfalo e superior da ponte, havendo uma certa seqüência topográfica, em estágios sucessivos cada vez mais profundos, de acordo com a intensidade do trauma. Assim, em pacientes vítimas de TCEs moderados, as LADs ocorrem na substância branca subcortical dos hemisférios cerebrais (estágio 1) (Figura 14). Nos pacientes com trauma mais severo, além das lesões na substância branca subcortical, também podem haver lesões na metade posterior do corpo caloso (estágio 2) (Figura 15). Nos pacientes com TCEs muito graves, além das lesões acima descritas, ocorrem lesões na região dorsolateral do mesencéfalo e porção superior da ponte (estágio 3) (Figura 16).

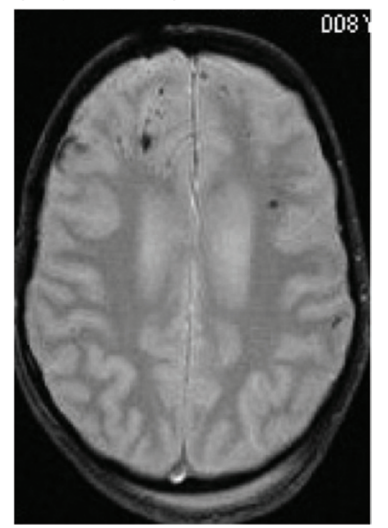

Figura 14. RM: LAD estágio 1: múltiplas lesões subcorticais hemorrágicas nos hemisférios cerebrais, evidenciadas na sequência T2* (também conhecida como gradiente eco)

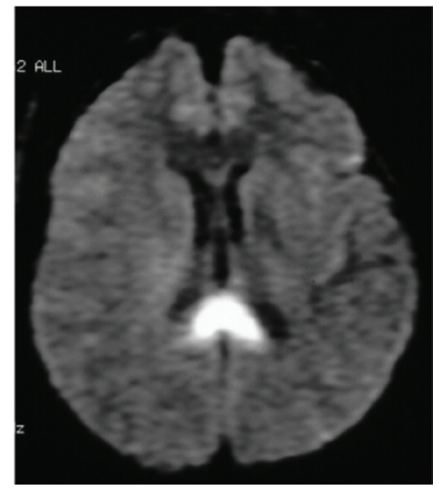

Figura 15. RM: LAD estágio 2: lesão com restrição da difusão das moléculas de água no esplênio do corpo caloso (imagem de difusão)

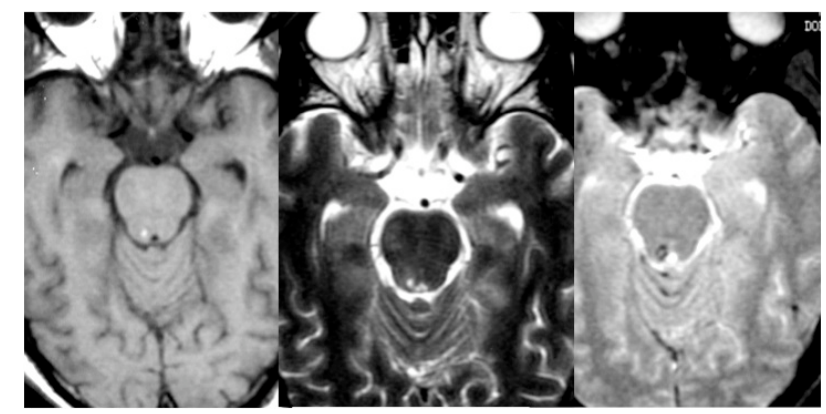

Figura 16. RM: LAD estágio 3: pequena lesão hemorrágica no teto mesencefálico, em região periaquedutal direita, na sequência pesada em T1 (imagem à esquerda), pesada em T2 (imagem ao centro) e T2* (gradiente eco) (imagem à direita)

$\mathrm{Na}$ TC, a LAD geralmente apresenta-se como múltiplas pequenas áreas ovaladas esparsas hemorrágicas ou hipoatenuantes, distribuídas nas localizações supracitadas (Figura 17), porém é método menos sensível que a RM, subestimando o grau de injúria, especialmente quando as lesões são profundas, pequenas ou não hemorrágicas. Assim, está indicada a RM quando há discrepância entre quadro clínico e os achados à $\mathrm{TC}^{3-7,19}$.

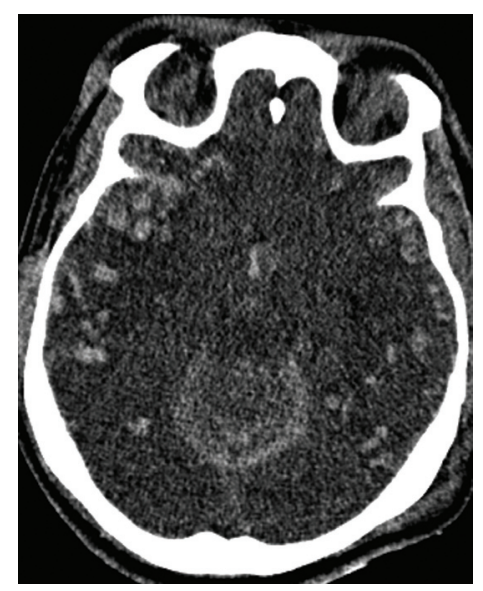

Figura 17. TC: LAD: múltiplas lesões subcorticais hemorrágicas hiperdensas nos hemisférios cerebrais

À RM, não há sequência de pulso específica e que seja a melhor para se diagnosticar LAD, entretanto, estudos mostram que a combinação de determinadas seqüências, cada qual com suas características, potencializam a detecção do maior número e volume de lesões. Assim, as lesões hemorrágicas são melhor detectadas pela sequência T2* gradiente eco e SWI (hipossinal), as lesões pequenas e não hemorrágicas, acompanhadas de edema vasogênico, são melhor detectadas pelas seqüências FLAIR e T2 "fast spin eco" (hipersinal), e finalmente, e ainda por mecanismos não completamente esclarecidos, a sequência de difusão contribui para a detecção de 
lesões (Figura 15). Assim, seqüências T2* gradiente eco, SWI, T2-Fast Spin Eco, FLAIR e difusão sempre devem fazer parte do protocolo de estudo por RM no paciente com TCE para melhor estadiar o diagnóstico de $L A D$, orientando conduta e estimando com mais precisão o prognóstico destes pacientes ${ }^{8-10}$.

Lesão de substância cinzenta subcortical: faz parte do espectro das lesões causadas por mecanismos traumáticos similares às LADs, havendo estiramento e laceração de vasos perfurantes, sendo caracterizada por múltiplas hemorragias petequiais primárias na substância cinzenta que é ricamente vascularizada, distribuídas preferencialmente nos núcleos da base, tálamos, regiões adjacentes ao III ventrículo e tronco cerebral. A TC é frequentemente normal devido à sua baixa sensibilidade na detecção deste tipo de lesão, mas eventualmente pode demonstrar focos hemorrágicos confluentes nos traumas mais graves (Figura 18), sendo a RM o método mais sensível, principalmente na detecção das lesões do tronco cerebral. Correspondem a $5 \%$ das lesões traumáticas encefálicas, sendo menos freqüente que a LAD e contusões corticais, e geralmente associadas a TCEs graves ${ }^{5,19}$.

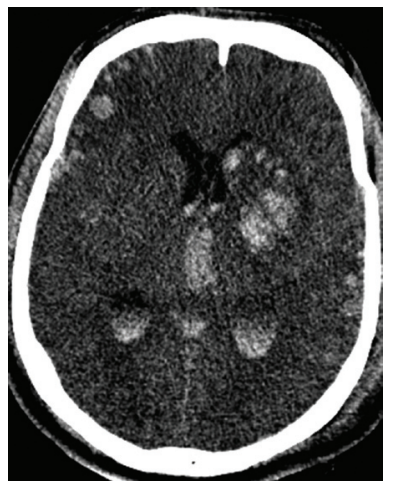

Figura 18. TC: Lesões hemorrágicas hiperdensas confluentes predominando na substância cinzenta profunda (núcleos da base) à esquerda e outras na transição corticosubcortical. Note ainda hemoventrículo

Contusão cortical: é uma das lesões mais freqüentemente encontradas em pacientes vítimas de TCEs graves. Estas lesões acometem a substância cinzenta cortical, podendo ou não haver comprometimento da substância branca subjacente, dependendo da intensidade do trauma. São causadas predominantemente por forças de aceleração/desaceleração que induzem um movimento inercial do encéfalo contra a tábua interna da calota craniana, sendo, por este motivo, mais freqüentemente encontradas nas regiões corticais adjacentes às estruturas ósseas irregulares da base do crânio explicando porque são mais freqüentes nas regiões basais, laterais e anteriores dos lobos temporais e frontais, e menos freqüentes nas regiões parietais, occipitais e cerebelares, frequentemente múltiplas e bilaterais. A movimentação do encéfalo durante o trauma pode produzir lesões corticais tanto no lado do golpe quanto no lado oposto ao golpe, caracterizando lesões em "golpe-contragolpe" (Figura 1). A substância cinzenta é mais vascularizada do que a substância branca, por isso as contusões corticais são mais hemorrágicas que as LADs. As contusões corticais, na sua fase aguda, geralmente mostram-se na TC como áreas corticais hipoatenuantes (componente não hemorrágico da contusão) podendo ou não conter zonas focais hiperatenuantes (componente hemorrágico agudo da contusão). Entre 24-48 horas, o componente hemorrágico hiperdenso tende a se expandir naquelas áreas contusionais inicialmente não hemorrágicas, podendo se estender às regiões subcorticais ou até mesmo comprometer grande parte de um ou mais lobos cerebrais, podendo apresentar evolução temporal desde pequenos focos hemorrágicos petequiais iniciais até extensas áreas hemorrágicas confluentes nos dias subsequentes. Nos hematomas contusionais maiores pode haver níveis líquidos intraparenquimatosos, sendo um achado

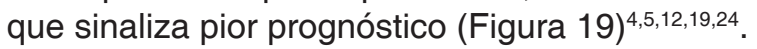

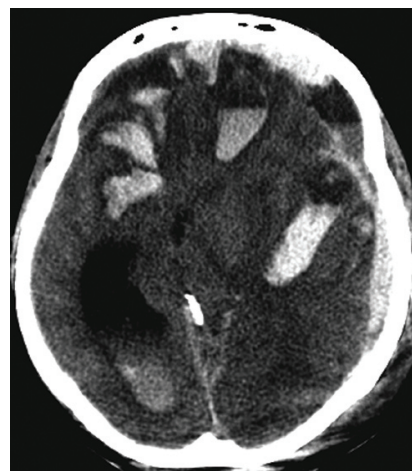

Figura 19. TC: TCE grave. Hematoma subdural agudo à esquerda, hemoventrículo e múltiplas e volumosas contusões corticais e subcorticais hemorrágicas hiperdensas formando níveis líquidos

\section{LESÕES TRAUMÁTICAS SECUNDÁRIAS}

As lesões traumáticas secundárias ocorrem em conseqüência a reações fisiológicas intracranianas ou sistêmicas em resposta à injúria inicial: neste grupo se incluem o edema, herniação, isquemia e infarto cerebrais, hemorragia secundária, infecção e hidrocefalia ${ }^{17}$.

Herniação cerebral: é o deslocamento de estruturas encefálicas de seu compartimento intracraniano original para o compartimento adjacente. As herniações cerebrais mais freqüentes são a subfalcina e a transtentorial descendente. A herniação subfalcina é caracterizada pelo deslocamento do giro do 
cíngulo insinuando-se por baixo da margem inferior da foice cerebral, ultrapassando a linha mediana. É identificada tanto na TC como na RM pelo desvio das estruturas de linha mediana (Figura 8). Quando muito acentuada, o ventrículo lateral ipsilateral apresentase comprimido, e o contralateral, alargado devido à compressão do forame de Monro. Pode ainda ocasionar deslocamento e compressão dos ramos da artéria cerebral anterior contra a foice, podendo ocasionar isquemia e infarto. A herniação transtentorial descendente, também denominada herniação uncal, é caracterizada pelo deslocamento medial do uncus e giro parahipocampal sobre a incisura da tenda cerebelar, comprimindo o tronco cerebral (Figuras 20 e 21). O deslocamento e compressão das artérias cerebrais posteriores podem ocasionar isquemia ou infarto occipital. Outras herniações menos freqüentes incluem a herniação transtentorial ascendente, tonsilar e transesfenoidal' ${ }^{17}$.

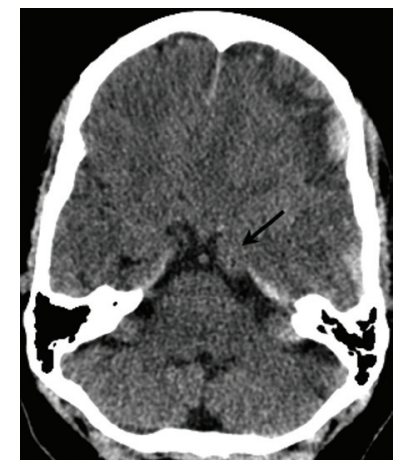

Figura 20. TC: TCE grave. Paciente com hematoma subdural agudo e contusões corticais à esquerda, mais bem demonstrados nos cortes tomográficos superiores, e neste nível determinando herniação uncal deste lado (seta)

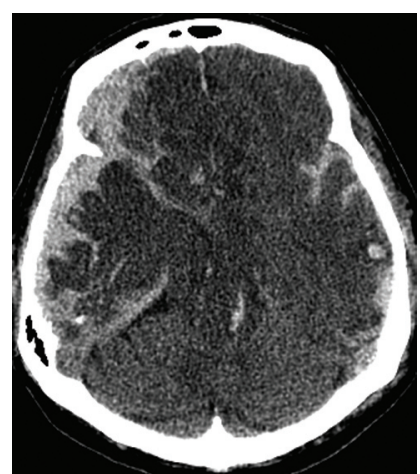

Figura 21. TC: Hematoma subdural agudo direito, hemorragia subaracnóidea aguda traumática, herniação uncal bilateral e hemorragia secundária (de Duret) caracterizada por faixas hemorrágicas hiperdensas nas porções centrais paramedianas da ponte

Infarto cerebral traumático secundário (ICTS) é causado pela compressão das estruturas vasculares principalmente na vigência de uma herniação cerebral. Estão presentes em 90\% dos TCEs fatais. O ICTS mais freqüente é o infarto occipital cau- sado pela compressão da artéria cerebral posterior numa herniação transtentorial descendente (uncal) (Figura 8). O segundo mais freqüente é o infarto em território da artéria cerebral anterior, geralmente causado por compressão do seu ramo calosomarginal devido a uma herniação subfalcina. Ambos mecanismos respondem por cerca de $80-90 \%$ dos ICTS. Infartos em território da artéria cerebral média podem ocorrer devido à herniação ou à edema cerebral severos. Menos freqüentemente, podem ocorrer infartos nos núcleos da base devido à compressão das artérias lenticuloestriadas, talamoperfurantes e coroidéias contra a base do crânio ${ }^{17}$. A RM permite o diagnóstico precoce do ICTS ao se demonstrar, na sequência de Difusão, restrição da movimentação das moléculas de água inferindo presença de edema citotóxico presente nos infartos agudos.

Hemorragia secundária: é causada principalmente pelas herniação transtentorial descendente. $O$ deslocamento caudal do mesencéfalo pode comprimir os vasos perfurantes na cisterna interpeduncular causando hemorragia no mesencéfalo (denominada hemorragia de Duret) ou eventualmente, isquemia. A hemorragia de Duret, de natureza secundária, ocorre predominantemente nas porções centrais do tronco cerebral, diferentemente da lesão primária do tronco cerebral também freqüentemente hemorrágica, mas que geralmente ocorre nas porções dorsolaterais. A hemorragia secundária pode ser detectada pela TC (Figura 21), mas por sua localização predominantemente infratentorial, é melhor estudada pela $\mathrm{RM}^{7,12}$.

Edema cerebral difuso ocorre pelo aumento do volume sanguíneo intravascular ou por aumento do conteúdo hídrico cerebral (edema propriamente dito), ou por ambos.

O aumento do volume sanguíneo intravascular ocorre por hiperemia, por vasodilatação decorrente da vasoplegia e perda da autorregulação vascular cerebral ou por congestão venosa. O aumento do conteúdo hídrico cerebral (edema propriamente dito), ocorre por diversos mecanismos que definem edema citotóxico, vasogênico, osmótico, hidrostático e hidrocefálico.

Seja qual for sua fisiopatologia, qualquer tipo de edema cerebral caracteriza-se por acúmulo de fluídos, principalmente água, seja no espaço intravascular, intracelular ou intersticial, produzindo aumento volumétrico encefálico em detrimento do espaço das vias de circulação liquórica. Ocorre em 10 a 20\% dos TCEs graves, sendo duas vezes mais freqüente em crianças do que em adultos. $O$ edema cerebral atinge um pico máximo de desenvolvimento entre 24 e 48 horas após o TCE. Sinais de herniações geralmente 
estão presentes, também podendo ocorrer compressões com estenoses ou oclusões vasculares que podem levar secundariamente a infartos.

A TC evidencia um apagamento difuso das cisternas e dos sulcos corticais, redução volumétrica do sistema ventricular e hipoatenuação difusa do parênquima cerebral com perda da diferenciação entre a substância branca e cinzenta (Figura 22). Quando o tronco cerebral e cerebelo estão poupados podese ter a falsa impressão de que sua atenuação está aumentada, na verdade sendo uma atenuação normal contrastando-se à acentuada hipoatenuação difusa dos hemisférios cerebrais ${ }^{17}$. A RM tem importância por poder diferenciar o edema citotóxico dos demais mecanismos de edema, pois qualquer edema irá apresentar hipossinal em T1 e hipersinal em T2 / FLAIR, mas somente o edema citotóxico apresenta redução da difusão das moléculas de água nas imagens de difusão e no respectivo mapa de coeficiente de difusão aparente. Assim, a RM demonstrando o mecanismo de edema predominante no paciente com TCE auxilia no seu manejo terapêutico e no seu prognóstico.

\section{REFERÊNCIAS}

1. Masters SJ. Evaluation of head trauma: efficacy of skull films. AJR Am J Roentgenol. 1980;135(3):53947.

2. Masters SJ, et al. Skull x-ray examinations after head trauma. Recommendations by a multidisciplinary panel and validation study. N Engl J Med. 1987;316(2):8491.

3. Gentry LR. Imaging of closed head injury. Radiology. 1994;191(1):1-17.

4. Gentry LR, et al. Prospective comparative study of intermediate-field MR and CT in the evaluation of closed head trauma. AJR Am J Roentgenol. 1988;150(3):673-82.

5. Gentry LR, Godersky JC, Thompson B. MR imaging of head trauma: review of the distribution and radiopathologic features of traumatic lesions. AJR Am J Roentgenol. 1988;150(3):663-72.

6. Gentry LR, Thompson B, Godersky JC. Trauma to the corpus callosum: MR features. AJNR Am J Neuroradiol. 1988;9(6):1129-38.

7. Gentry LR, Godersky JC, Thompson BH. Traumatic brain stem injury: MR imaging. Radiology. 1989;171(1):17787.

8. Ezaki $Y$, et al. Role of diffusion-weighted magnetic

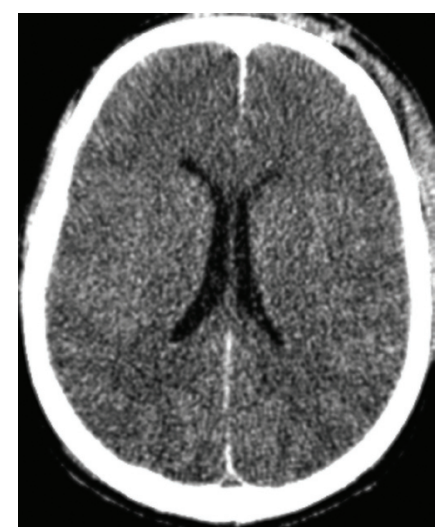

Figura 22. TC: Edema cerebral difuso com apagamento dos sulcos corticais e má definição entre substância branca e cinzenta cerebrais. Há sinais de hemorragia subaracnóide junto à foice cerebral

\section{CONCLUSOES}

Os métodos de imagem (TC e RM) fornecem importantes informações diagnosticas e prognósticas nos pacientes com TCE.

resonance imaging in diffuse axonal injury. Acta Radiol. 2006;47(7):733-40.

9. Huisman TA. Diffusion-weighted imaging: basic concepts and application in cerebral stroke and head trauma. Eur Radiol. 2003;13(10):2283-97.

10. Schaefer PW, et al. Diffusion-weighted MR imaging in closed head injury: high correlation with initial glasgow coma scale score and score on modified Rankin scale at discharge. Radiology. 2004;233(1):58-66.

11. Provenzale J. CT and MR imaging of acute cranial trauma. Emerg Radiol. 2007;14(1):1-12.

12. Udstuen GJ, Claar JM. Imaging of acute head injury in the adult. Semin Ultrasound CT MR. 2001;22(2):135-47.

13. Thornbury JR, et al. Skull fracture and the low risk of intracranial sequelae in minor head trauma. AJR Am J Roentgenol. 1984;143(3):661-4.

14. Osborn AG. Diagnostic neurorradiology. St Louis: Mosby; 1994. p.199-247.

15. Martins RS, et al. Prognostic factors and treatment of penetrating gunshot wounds to the head. Surg Neurol. 2003;60(2):98-104; discussion 104.

16. Jinkins JR, et al. Value of acute-phase angiography in the detection of vascular injuries caused by gunshot wounds to the head: analysis of 12 cases. AJR Am J 
Roentgenol. 1992;159(2):365-8.

17. Osborn AG. Diagnostic imaging. Brain. Salt Lake City: Amirsys; 2004. p.4-63, v. Section 2 - Trauma.

18. Zimmerman RA, Bilaniuk LT. Computed tomographic staging of traumatic epidural bleeding. Radiology. 1982;144(4):809-12.

19. Atlas SW. Magnetic resonance imaging of the brain and spine. Philadelphia: Lippincot-Raven; 1996. p.611-47.

20. Server A, et al. Vertex epidural hematoma - neuroradiological findings and management. Acta Radiol. 2002;43(5):483-5.

21. Harbury OL, Provenzale JM, Barboriak DP. Vertex epidural hematomas: imaging findings and diagnostic pitfalls. Eur J Radiol. 2000;36(3):150-7.

22. Ball WS Jr. Nonaccidental craniocerebral trauma (child abuse): MR imaging. Radiology. 1989;173(3):60910.

23. Cohen RA, et al. Cranial computed tomography in the abused child with head injury. AJR Am J Roentgenol.
1986;146(1):97-102.

24. Grossman RIY, Yousem DM. Neuroradiology: the requisites. St. Louis, MO: Mosby; 1994. p.149-69.

25. Ommaya AK, Goldsmith W, Thibault L. Biomechanics and neuropathology of adult and paediatric head injury. Br J Neurosurg. 2002;16(3):220-42.

26. Shetty PG, et al. Evaluation of high-resolution CT and MR cisternography in the diagnosis of cerebrospinal fluid fistula. AJNR Am J Neuroradiol. 1998;19(4):633-9.

27. Reed $D$, et al. Acute subdural hematomas: atypical CT findings. AJNR Am J Neuroradiol. 1986;7(3):41721.

28. Boyko OB, Cooper DF, Grossman CB. Contrastenhanced CT of acute isodense subdural hematoma. AJNR Am J Neuroradiol. 1991;12(2):341-3.

29. Stein SC, et al. Delayed brain injury after head trauma: significance of coagulopathy. Neurosurgery. 1992;30(2):160-5.

30. Huisman TA. Intracranial hemorrhage: ultrasound, CT and MRI findings. Eur Radiol. 2005;15(3):434-40. 